\title{
MAL DE ALTURA
}

\author{
Dr. MARCELO MOREANO D.
}

Servicio de Cardiología. Clínica Sta. Cecilia, Quito

Ya el profesor Hurtado en 1937 lo califica como un fenómeno bastante frecuente en personas jóvenes que viven en la altura y que retornan a ella luego de períodos relativamente cortos de tiempo de permanencia a nivel del $\operatorname{mar}^{1}$. Constituye una emergencia respiratoria y cardíaca con manifestaciones de insuficiencia de la primera $y$ sobrecargada derecha de la segunda. Aparece en forma aguda, violenta y progresiva, no conocemos de casos que hayan revertido espontáneamente, pero sí de medidas preventivas que lo haya evitado. Es susceptible de aparecer desde las primeras horas de haber retornado a la altura y casos se han descrito en que lo han hecho hasta 72 horas más tarde ${ }^{2}$. Lo hemos visto y tratado en niños de dos años de edad hasta adultos de 27 años. Nuestra causística, igual que en causísticas extranjeras, es de franco predominio en el hombre.

Los episodios iniciales en todos nuestros pacientes se presentaron luego de actividad física más o menos intensa y prolongada; no obstante, se han descrito casos en los cuales se ha presentado durante el sueño como el descrito por Peñaloza y Sime?
ETIOLOGIA, FISIOPATOGENIA Y PATOGENIA.- Todos los autores que se han ocupado del estudio del mal de altura coinciden en que la hipoxia es el factor fundamental en la Etiología de este fenómeno. Marticorena admite que si bien no existe hipertensión capilar pulmonar en estos pacientes es inevitable la presencia o incremento de la permeabilidad capilar para así justificar los hallazgos evidentes del cuadro clínico de edema pulmonar ${ }^{4}$, sin embargo ésto, que clínicamente constituye un hecho, no ha poidido ser demostrado en experimentos en hipoxia aguda; la presencia de fibrina y membrana hialina en el exudado alveolar de estos casos parece ser el mejor argumento a favor del incremento de la permeabilidad capilar.

Aceptada la hipoxia como factor fundamental del problema, ésta determina intenso vasoespasmo arterial precapilar con repercusión hipertensiva arterial, pulmonar, permeabilización de la red arteriolar precapilar, congestión, edema y trasudación. Fred ${ }^{5}$ y Hultgren y $\operatorname{col}^{6}$ fueron los primeros en llamar la atención en el sentido de excluír por completo la posibilidad de una claudi- 
cación ventricular izquierda o de una repercusión vasoespástica venosa pulmonar cuando encontraron que las presiones capilares pulmonares registradas en pleno episoidio agudo se encontraban dentro de límites normales.

Debemos mencionar que existen también otros factores que se suman a los descritos en la producción de este fenómeno, así por ejemplo Fritts y Cournand" comprobaron que "el gasto cardíaco se incrementa con la hipoxia aguda experimental y que la magnitud de este incremento se relaciona con el grado de anoxemia". Hurtado y Aste Salazar demostraron que el grado de anoxemia es mucho más acentuado en aquellos que viviendo en la altura no son nativos de ella ${ }^{8}$, mientras que el gasto cardíaco permanece normal en los residentes en la altura, como lo demuestra Peñaloza y $\mathrm{col}^{9}$. Así pues es lógico suponer que el edema pulmonar se producirá luego del ejercicio en la altura pues con éste se incrementa el gasto cardíaco. Finalmente es interesante que esta patología se produzca durante el sueño pues en éste existiría un incremento del gasto cardíaco por cambios postulares y al mismo tiempo una caída en la saturación de oxígeno en el decúbito dorsal, como la analiza Marticorena ${ }^{4}$.

Parece también que existe un incremento en el volumen sanguíneo pulmonar; la baja temperatura de las altitudes, la palidez y el frío con que responde el individuo sugieren la presencia de una vasoconstricción periférica significativa. Sarnof se inclina en este sentido $^{10}$ y es importante considerar que el incremento del volumen sanguíneo pulmón preceda a la producción del edema. Asimismo no perdamos de vista los criterios de Marticorena y Reinafarge y col. ${ }^{11}$ en el sentido de que los nativos de altura que presentaban este fenómeno pierden parcialmente sus mecanismos de adaptación, pues el volumen sanguíneo total característicamente incrementado en estos sujetos no decrece durante las primeras semanas de residencia a nivel del mar, ya que la menor proiducción de eritrocitos está compensada por el incremento proporcional en volumen plasmático.

Se ha hablado también de una predisposición individual que no representaría sino un incremento desproporcional de los fenómenos fisio-patológicos normales en individuos que retornamos a nuestros lugares de origen, luego de visitar estas saludables y bien oxigenadas áreas de nuestra patria (factor familiar).

Finalmente algo debemos decir sobre la importancia de factores humorales, cuya liberación como producto de la hipoxia incrementan los fenómenos de permeabilidaid capilar, edema y trasudación, es el caso de la serotonina en cuyo estudio estamos interesados.

SINTOMATOLOGIA. - Fernando Tapia $^{12}$ la describe muy bien resumiéndola en esta forma "los síntomas iniciales más frecuentes fueron: cefalea, intranquilidad, disnea progresiva, tos seca ,palpitaciones y náusea. En el episodio agudo disnea marcada, tos con expectoración espumosa, blanquecina o rosada, sudoración fría, ansiedad y ocasionalmente vómito, escalofrío, dolores 
ósteo-articelulares difusos, sed, debilidad general y trastornos sensoriales. En el examen físico disnea ortopnea, cianosis labial y digital, cara pálida, piel fría y húmeda. Se escucharon abundantes estertores húmedos (finos y gruesos) y roncus en ambos campos pulmonares; taquicardia y discreta o moderada hipotensión. Fielbre hasta de 1 . C.

Nosotros hemos observado en los 21 casos que hemos tenido oportunidad de controlar, que no es posible hablar de una sintomatología única, que si bien los síntomas y los signos se los puede enumerar en forma más o menos idéntica, sin embargo clínicamente hablando puede decirse que existen 4 estudios evolutivos y a los cuales no debe perdérselos de vista, pues en forma categórica afirmamos que tanto más benigna es la evolución, cuánto más precozmente se la diagnostica y se la trata.

La primera modalidad o estadio, corresponde a aquellos individuos que tan sólo se quejan de discreta disnea, cefalea, mareo, pesantez cefálica, astenia y que en el examen físico puede o no estar presente discreta cantidad de rales de medianas y finas burbujas en uno o más segmentos pulmonares.

La segunda modalidad o segundo estado corresponde a la sintomatología de disnea, de moderados esfuerzos, ortopnea ocasional, cefalea llamativa y persistente, náusea, palpitaciones precordialles, astenia y malestar general. En el examen físico ya se encuentran moderada polipnea, dificultad en el decúbito dorsal completo, palidez, cianosis mínima, ralles y roncus y ocasionales sibilancias diseminadas en uno o ambos campos pulmonares. Taquicardia.

La tercera modalidad o tercer estadio ya se caracteriza por presencia de la sintomatología anterior sobre añadida de tos y expectoración espumosa, blanquecina y rosácea, abundante, persistente, notablemente flúida. Disnea contínua, ortopnea permanente, cianosis intensa, notable angustia, ansiedad, dolores musculares, sensación de agotamiento físico. En el examen físico la cianosis es inequívoca, la respiración superficial y taquipneica, la palidez cianótica es llamativa y cuando se une a sudoración fría y persistente el cuadro es inconfundible. No hay mayor variante en la presión arterial aunque la taquípnea y la taquicardia son considerables. Fiebre.

La cuarta modalidad la hemos visto en dos ocasiones, en muchachos de 17 y 21 años respectivamente, los dos consultaron al iniciarse el tercer día de su evolución clínica, los dos fueron tratados en forma equivocada inicial. Las manifestaciones descritas estaban presentes todias en grado máximo, los enfermos estuvieron estuporosos, semiinconscientes, hipotensos, exageradamente cianóticos a pesar de permanecer en tienda de oxígeno. Fiebre de $39^{\circ}$ C. El curso fue progresivo y fatal dentro de las ocho primeras horas de tratamienito. En los dos llamó la atención notablemente el hecho de la expulsión masiva de líquido seroso en el primero y sero-sanguinolento en el segundo, por las vías respiratorias al momento de su expiración. Cabe decir que los pacien- 
tes murieron ahogados en sus propias secreciones.

EXAMENES COMPLEMENTARIOS.- En nuestra causística éstos guardan íntima relación con las modalidades descritas, así la leucocitosis, la desviación izquierda de la fórmula, la poliglobulia, el salpicado congestivo pulmonar, las modificaciones electrocardiográficas de tipo sobrecarga e hipertrofia derecha, todas guardaron relación con sintomatología.

Dos estudios hemodinámicos realizados demostraron normalidad.

La EVOLUCION asimismo clínicamente se relaciona con cada una de las moidalidades; en las tres primeras, muy satisfactoria y con restitución ad-integrum, en la cuarta con éxito letal.

COIPLICACIONES. - Las hemos visto de la siguiente naturaleza:

1) Con insuficiencia cardíaca derecha de la modalidad tres, en dos casos.

2) Con manifestaciones residuales de tipo infeccioso (febrículo persistente, expectoración muco-purulenta de cuarto o quinto día, roncus y estertores tráqueo laríngeos).

3) Neumotórax y enfisema mediastinal descritos por Arcos y coll. ${ }^{13}$ y en cuya causística se invollucró uno de nuestros casos, y

4) Muerte.

TRATAMIENTO. - Por sistema insistimos en la prevención, que la podemos resumir en reposo absoluto en cama inmediato al regreso a la altura desde el nivel del mar y viaje de preferencia en avión.

Clínicamente para la primera moda- lidad aconsejamos reposo físico y oxígenoterapia.

Para la segunda modalidad, reposo físico, oxígenoterapia y moderada sedación.

Para la tercera modalidaid reposo físico absoluto, oxígenoterapia en atmósfera que tenga el $100 \%$ de saturación, intensa sedación de preferencia con morfina. Antiserotonínicos. Potencialmente digitálicos.

Para la cuarta, todas las medidas anteriores, antibióticos, sangría, acetilcolina (como vasodilatador pulmonar), máximo cuidado en la permeabilidad de las vías respiratorias y como terapia inespecífica corticoesterolides (stress?).

En una de nuestras defunciones se realizó el examen necrópsico, el grado de hepatización pulmonar fue severísimo, puede afirmarse que los dos campos pulmonares estaban toitalmente bloqueados, el ventrículo derecho y la arteria pulmonar estuvieron dilatadas. Existió hepatomegalia congestiva y discreta esplenomegalia.

Séame permitido señalar los hallazgos de Marticorena ${ }^{4}$ en cuanto a las características microscópicas: "extensas áreas con membranas hialinas en estrecho contacto con la pared alveolar, asimismo material fibrinoso mayor en los lóbulos medios e inferiores. El análisis histoquímico de la membrana hialina reveló una idéntica composición a la diel recién nacido. Los septum alveolares fueron gruesos debido a la marcada congestión capilar. Areas focales de enfisema alternaban con áreas de edema. Ambos casos presentaron en- 
grosamiento de la capa muscular arterial, de numerosas arteriolas terminales y vasos interseptales. Estos cambios fueron más severos que aquellos observados en individuos aclimatizados residentes en la altura.

\section{BIBLIOGRAFIA}

1. HURTADO, A.: Aspectos fisiológicos de la vida en la altura. Imp. Edit. Rimac S. A., 1937.

2. PEÑALOZA, D. y col.: Symposium sobre: El corazón y la circulación pulmonar en los habitantes de las grandes alturas. Revista del Viernes médico, $\mathbf{X V}$ : $64,1964$.

3. PENTALOZA, D. y col.: Pulmonary circulation during high altitude. Pulmonary edema. Bulletin de Physio-Pathologie Respiratoire, 4: 1963.

4. MARTICORENA, E.: Pulmonary edema by adcending to high altitudes. Diseases of the Chest., 45: 273, 1964.

5. FRED, H. L. y col.: Acute pulmonary edema of Altitude, Circulation, 25: 929, 1962.

6. HULTGREN, H. N. y col.: Further Studies of High Altitude Pulmonary Edema. Brit. Heart. J., 24: 95, 1962.

7. FRITTS, H. W. y col.: Physiological Factors Regulating Pressure, Flow and
Distribution of Blood in the Pulmonary Circulation, In: Pulmonary. Circulation, 62, 1959.

8. HURTADO, A. y col.: Arterial Blood Gases and Acid-Base Balance al Sea Level and at High Altitudes. J. Appl. Physiol, 1: 304, 1948.

9. PEÑALOZA, D. y col.: Pulmonary Hipertension in Healthy Men Born and Living at High Altitudes. Am. J. Cardiol, 11: $150,1963$.

10. SARNOFF, S. y col.: Neuro-hemodynamics of Pulmonary Edema. II. The role of Symoathetic Pathways in the Elevation of Pulmonary and Systemic Vascular Pressures Following the Intracisternal Injection of Fibrin Circulation, 6: 51, 1952.

11. REYNAFARJE, C. y col.: The Polycythemia of High Altitudes. Iron Metabolism and Related Aspects. Blood, 14: 433, 1959.

12. TAPIA, F.: Symposium sobre. Ell corazón y la circulación pulmonar en los habitantes de las grandes alturas. Segunda sesión. Aspectos fisiológicos y clínicos. Edema pulmonar por ascención a las grandes alturas. Trabajo presentado en la 165\% reunión del viernes Médico. Lima.

13. ARCOS, L. y col.: Enfisema Mediastínico y Neumotorax en Edema pulmonar de Altura. Revista Ecuatoriana de Medicina y Ciencias Biológicas, IV: 1966. 